Quim. Nova, Vol. 33, No. 3, 634-638, 2010

\title{
PERFIL QUÍMICO DE CULTIVARES DE FEIJÃO (Phaseolus vulgaris) PELA TÉCNICA DE HIGH RESOLUTION MAGIC ANGLE SPINNING (HR-MAS)
}

\author{
Luciano Morais Lião*, Rafael Choze, Pedro Paulo Araujo Cavalcante, Suzana da Costa Santos e Pedro Henrique Ferri \\ Instituto de Química, Universidade Federal de Goiás, CP 131, 74001-970 Goiânia - GO, Brasil \\ Antonio Gilberto Ferreira \\ Departamento de Química, Universidade Federal de São Carlos, CP 676, 13565-905 São Carlos - SP, Brasil
}

Recebido em 29/4/09; aceito em 3/9/09; publicado na web 12/2/10

\begin{abstract}
CHEMICAL PROFILE OF BEANS CULTIVARS (Phaseolus vulgaris) BY ${ }^{1} \mathrm{H}$ NMR - HIGH RESOLUTION MAGIC ANGLE SPINNING (HR-MAS). The application of one-dimensional proton high-resolution magic angle spinning ( ${ }^{1} \mathrm{H}$ HR-MAS) NMR combined with a typical advantages of solid and liquid-state NMR techniques was used as input variables for the multivariate statistical analysis. In this paper, different cultivars of beans (Phaseolus vulgaris) developed and in development by Embrapa - Arroz e Feijão were analyzed by ${ }^{1} \mathrm{H}$ HR-MAS, which have been demonstrated to be a valuable tool in its differentiation according chemical composition and avoid the manipulation of the samples as used in other techniques.
\end{abstract}

Keywords: ${ }^{1} \mathrm{H}$ HR-MAS NMR technique; Phaseolus vulgaris; multivariate statistical analysis.

\section{INTRODUÇÃO}

A ressonância magnética nuclear tem sido uma técnica muito útil na análise de materiais sólidos onde a preparação da amostra, na maioria das vezes, se resume ao seu empacotamento no rotor. Entretanto, no estado sólido as fortes interações dipolares são a principal causa do alargamento dos sinais, podendo alcançar valores da ordem de $\mathrm{kHz}$ e são decorrentes das interações diretas e através do espaço dos núcleos presentes nas moléculas, devido a grande restrição dos movimentos moleculares quando comparado com os sistemas em solução, onde os movimentos Brownianos rápidos fazem com que essas interações sejam praticamente nulas. Por outro lado, os efeitos de anisotropia do deslocamento químico, originário devido ao fato dos núcleos sentirem campos magnéticos locais $\left(\mathrm{B}_{\text {local }}\right)$ diferentes de $\mathrm{B}_{1}$, uma vez que existe o efeito de proteção/desproteção magnética dos núcleos devido ao campo magnético provocado pelas nuvens eletrônicas, são sobrepujados em muito pelas interações dipolares. A utilização de técnicas combinadas, como a rotação no ângulo mágico e o desacoplamento de alta potência, minimiza drasticamente os efeitos de alargamento de sinais observados nos espectros de materiais sólidos. ${ }^{1}$

Apesar do grande avanço no estudo de RMN de amostras sólidas, tradicionalmente não se tem explorado a $\mathrm{RMN}$ de ${ }^{1} \mathrm{H}$, uma vez que as interações moleculares ainda comprometem significativamente a resolução espectral, no nível de separação que exige um espectro de hidrogênio. Nesse contexto, a técnica High Resolution Magic Angle Spinning - HR-MAS tem demonstrado ser bastante promissora no estudo de sólidos heterogêneos. ${ }^{2}$ Através da HR-MAS, têm sido estudados materiais intactos de vegetais, como folhas, frutos e flores, ${ }^{3}$ e de animais, como fígado, cérebro, pulmão, rim, entre outros. ${ }^{4} \mathrm{~A}$ aplicação dessa técnica tem apresentado ainda excelentes resultados na caracterização de géis, ${ }^{5}$ moléculas adsorvidas em zeólitas ${ }^{6}$ e ligadas a resinas, ${ }^{7}$ proteínas nanocristalinas, ${ }^{8}$ micro-organismos intactos ${ }^{9} \mathrm{e}$, recentemente, aplicada a nanopartículas de óxido de ferro - contendo ferro paramagnético, onde demonstrou ser possível a obtenção de espectros com sinais finos e bem resolvidos. ${ }^{10} \mathrm{Na}$ quimiotaxonomia de liquens, essa técnica demonstrou ser uma relevante ferramenta na

\footnotetext{
*e-mail: luciano@quimica.ufg.br
}

discriminação entre famílias, gêneros e espécies, onde a identificação, baseada somente na análise morfológica, é comumente complexa e pouco conclusiva. ${ }^{11}$ Vale destacar que a técnica HR-MAS é muito sensível e requer poucos minutos para o registro de sinais de RMN de ${ }^{1} \mathrm{H}$ utilizando alguns miligramas do analito.

A técnica de RMN HR-MAS utiliza-se do fato de que os materiais, tanto vegetais quanto animais, quando no seu estado natural possuem moléculas de água em suas células na quantidade necessária para proporcionar mobilidade dos metabólitos (primários e secundários) e permitir a obtenção de espectros com excelente resolução, necessitando apenas da adição de uma ou duas gotas de solvente deuterado, adequadamente escolhido para não causar dissolução de parte dos constituintes das amostras - normalmente utiliza-se $\mathrm{D}_{2} \mathrm{O}$ para o ajuste da homogeneidade do campo magnético (shimming). Nesse caso, o material é fracionado em pequenos pedaços e inserido em um rotor com um volume esférico de 12 ou $50 \mu \mathrm{L}$. Por outro lado, quando a amostra já se encontra seca, pulveriza-se o material e adiciona-se $\mathrm{D}_{2} \mathrm{O}$ com os seguintes propósitos: hidratação do material e ajuste da homogeneidade do campo magnético. A geometria interna esférica do rotor aliada ao giro segundo o ângulo mágico de $54,74^{\circ}$, com uma rotação rápida - normalmente de 5-6 KHz, dependendo do campo magnético estático, permite se obter, por exemplo, espectros de hidrogênio de um material semi-sólido (ou heterogêneo), com a resolução similar ao de um espectro em solução. Assim, os problemas referentes aos acoplamentos dipolares, anisotropia do deslocamento químico e suscetibilidade magnética, sendo essa em muito minimizada pela geometria interna do rotor, são enormemente reduzidos. Isso pode ser observado na Figura 1, onde se pode comparar os espectros de RMN de ${ }^{1} \mathrm{H}$ de um material semi-sólido (heterogêneo) - folhas de Citrus limonea, utilizando um rotor de uso comum para amostras no estado sólido - CPMAS; um rotor com geometria interna especial - utilizado para amostras semi-sólidas, ambos girando com a mesma velocidade e no ângulo mágico e, ainda, o espectro do extrato hidroalcoólico do mesmo material em solução de $\mathrm{D}_{2} \mathrm{O}$. Como se pode observar, a resolução do espectro em solução é muito semelhante à obtida do material semi-sólido na sonda de HR-MAS utilizando-se o rotor com geometria interna esférica. Na Figura 1, apresenta-se, ainda, o espectro desse material semi-sólido obtido em uma sonda de sólidos - CPMAS, utilizando um rotor de geometria interna es- 
férica e observa-se uma resolução inferior àquela apresentada pela sonda HR-MAS. Além disso, outros experimentos unidimensionais, como RMN de ${ }^{13} \mathrm{C}\left\{{ }^{1} \mathrm{H}\right\}$, DEPT135 e bidimensionais como gCOSY, gHSQC, gHMBC, DOSY, entre outros, podem ser obtidos através da sonda de HR-MAS utilizando-se as mesmas sequências de pulsos que são utilizadas rotineiramente para a obtenção de espectros em solução. Obtendo-se espectros de RMN de ${ }^{1} \mathrm{H}$ com boa resolução, os mesmos podem ser utilizados como fingerprinting, sendo possível observar diferenças sutis entre as várias amostras, sem a necessidade de qualquer procedimento específico para extração e purificação das substâncias para posterior análise.

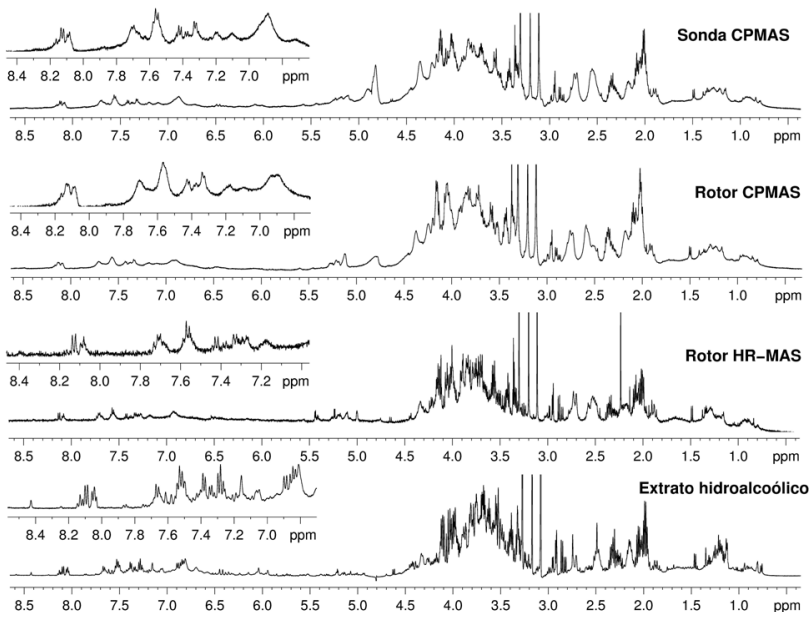

Figura 1. Espectros de $R M N$ de ${ }^{1} H$ de folhas de laranjeira no estado semisólido (heterogêneo) em uma sonda CPMAS e espectros de RMN HR-MAS utilizando-se um rotor de CPMAS $(80 \mu L)$, um rotor de HRMAS $(12 \mu L)$ e do extrato hidroalcoólico obtido em solução de $\mathrm{D}_{2} \mathrm{O}$

O uso de materiais intactos é um dos motivos pelos quais a RMN tem sido cada vez mais utilizada na análise de alimentos, uma vez que minimiza o tempo de análise e evita o pré-tratamento das amostras, comumente realizado em outras técnicas analíticas, que podem causar alterações na composição química do material em análise. Como exemplo, Gil e colaboradores visando acompanhar as mudanças na composição do fruto da mangueira (Mangifera indica L.) durante o seu amadurecimento, aplicaram a técnica HR-MAS em conjunto com a análise de RMN de líquidos e identificaram diversos constituintes, como ácidos, aminoácidos e compostos minoritários, além de determinarem as principais diferenças causadas pelo amadurecimento da fruta. ${ }^{3} \mathrm{~A}$ aplicação dessa técnica em amostras de carne, farinha de trigo e pão resultou em informações qualitativas e quantitativas que foram utilizadas na caracterização e classificação das diferentes amostras. ${ }^{12} \mathrm{Em}$ trabalho anterior, Sacco e colaboradores também analisando farinha de trigo de diferentes regiões do sul da Itália, além de obterem informações sobre os constituintes químicos das amostras, verificaram tendências de discriminação quanto à origem geográfica das mesmas. ${ }^{13} \mathrm{~A}$ técnica HR-MAS demonstrou ainda ser uma valiosa ferramenta no controle de qualidade de queijos, fornecendo informações sobre a caracterização organoléptica, origem e idade das amostras. ${ }^{14}$ Vale ressaltar que o poder e a eficiência das análises por RMN podem ainda ser intensificados pela aplicação dos métodos de análise multivariada, ou seja, métodos quimiométricos, cuja aplicação reduz a complexidade dos dados e evidencia as informações mais importantes. ${ }^{15}$

Neste artigo descreve-se a aplicação da espectroscopia de RMN de ${ }^{1} \mathrm{H}$, empregando em especial a técnica de HR-MAS, para a análise de cultivares do feijão comum desenvolvidos e em desenvolvimento na Embrapa - Arroz e Feijão, de Goiânia, Goiás.
O feijão comum, Phaseolus vulgaris L. (Fabaceae), é uma das mais importantes fontes de proteínas, fibras alimentares, ferro, carboidratos complexos, minerais e vitaminas para milhões de pessoas em países desenvolvidos e em desenvolvimento. O Brasil tem uma produção anual de cerca de 3,5 milhões de toneladas do feijão em grão, sendo que o consumo anual é, em média, de $18 \mathrm{~kg}$ por habitante. Somente no estado de Goiás são produzidas anualmente cerca de $268.000 \mathrm{t}$ de feijão, representando uma produtividade de $2.013 \mathrm{~kg}$ / ha, o que está acima da média nacional $(856 \mathrm{~kg} / \mathrm{ha})$ e, portanto, uma lavoura muito promissora no Estado. ${ }^{16}$

Além de ser uma fonte de nutrientes essenciais, o feijão possui constituintes químicos que são considerados componentes com ação funcional, como os flavonoides, incluindo flavonóis, glicosilados ou não, antocianidinas, proantocianidinas e isoflavonas, bem como ácidos fenólicos. ${ }^{17}$ Alimento funcional é um "alimento convencional ou similar a este em aparência, consumido como parte da dieta normal, que apresenta efeitos fisiológicos benéficos e/ou reduz o risco de doenças crônicas, além de suas funções nutricionais básicas". ${ }^{18}$ Estudos realizados com diversas variedades de feijão demonstraram o seu potencial como alimento funcional, devido a sua ação na diminuição dos riscos de doenças cardiovasculares e renais $;{ }^{19}$ redução no índice glicêmico para portadores de diabetes ${ }^{20}$ aumento na saciedade ${ }^{21}$ e na prevenção do câncer. ${ }^{22}$ Gibson e Roberfroid ${ }^{23}$ demonstraram a importância de oligossacarídeos e, dentre esses, a rafinose e a estaquiose que estão presentes no feijão como constituintes funcionais alterando favoravelmente a flora bacteriana. Por outro lado, devido à ausência de $\alpha$-galactosidase em humanos, esses oligossacarídeos são fermentados anaerobicamente pela microflora intestinal produzindo dióxido de carbono, hidrogênio e pequenas quantidades de metano, causando flatulência e desconfortos abdominais. O cozimento do feijão, principalmente sob altas pressões, é uma alternativa para a redução significativa dos teores desses açúcares. ${ }^{24}$

\section{PARTE EXPERIMENTAL}

\section{Coleta do material e preparo das amostras}

Doze cultivares distribuídos em seis variedades de feijão comum (Tabela 1) foram coletados na Estação Experimental da Embrapa Arroz e Feijão, Goiânia, GO.

Os grãos foram moídos em moinhos de facas, com adição de nitrogênio líquido a fim de se obter um pulverizado de baixa granulometria e com a menor quantidade de água possível.

Tabela 1. Cultivares e variedades (grupo) de Phaseolus vulgaris selecionados na Estação Experimental da Embrapa Arroz e Feijão, Goiânia, GO

\begin{tabular}{lccccc}
\hline Código & Cultivar & Grupo & Código & Cultivar & Grupo \\
\hline 1 & Jalo precoce & \multirow{2}{*}{ Jalo } & $\mathbf{7}$ & Valente & \multirow{2}{*}{ Preto } \\
$\mathbf{2}$ & Jalo EEP & & 8 & Campeiro & \\
3 & Radiante & & $\mathbf{9}$ & Pitanga & Roxinho \\
$\mathbf{4}$ & Irai & Rajado & 10 & Timbó & \\
5 & Pérola & & $\mathbf{1 1}$ & Marfim & Mulatinho \\
$\mathbf{6}$ & Requinte & Carioca & 12 & Corrente & \\
\hline
\end{tabular}

\section{Medidas espectroscópicas}

Os espectros de RMN de ${ }^{1} \mathrm{H}$ foram obtidos nos Laboratórios de RMN do DQ-UFSCar e IQ-UFG utilizando, respectivamente, um espectrômetro Bruker Avance DRX 400 de 9.4 T (400,21 MHz para a frequência do $\left.{ }^{1} \mathrm{H}\right)$ e um Bruker Avance III 500 de 11.75 T (500,13 $\mathrm{MHz}$ para o ${ }^{1} \mathrm{H}$ ), equipados com sondas HR-MAS de $4 \mathrm{~mm}$, com 
gradiente de campo na direção z. O material vegetal pulverizado e 2 gotas de uma solução de trimetilsililpropionato de sódio (TMSP$\left.2,2,3,3-d_{4}\right)$ em $\mathrm{D}_{2} \mathrm{O}(0,1 \% \mathrm{~m} / \mathrm{v})$ foram acondicionadas em um rotor de zircônio de $12 \mu \mathrm{L}$. Nesse caso, o solvente deuterado foi usado para o ajuste da homogeneidade do campo magnético, lock do equipamento e para proporcionar maior mobilidade das moléculas nas amostras. Os espectros foram realizados em triplicata, com uma velocidade de giro de $5 \mathrm{kHz}$, sem controle de temperatura, com uma largura espectral de $5593 \mathrm{~Hz}, 32 \mathrm{k}$ de pontos digitalizados e acúmulo de 192 FIDs. O processamento foi feito com $32 \mathrm{~K}$ pontos e utilizou-se uma multiplicação exponencial com lb (line broadening) $=0,3 \mathrm{~Hz}$.

Várias sequências de pulsos foram avaliadas para a supressão do sinal da água, destacando-se as sequências CPMG (Car-PurcellMeiboom-Gill) modificada e ZGCPPR (pré- saturação do sinal do solvente por pulsos compostos). A primeira utiliza uma pré-saturação do sinal da água com onda contínua seguida de uma sequência CPMG modificada, funcionando como um filtro de $\mathrm{T}_{2}$, para eliminar os sinais das moléculas com tempo de relaxação transversal $\left(\mathrm{T}_{2}\right)$ curto e possibilitar uma melhor visualização dos sinais de moléculas com baixa massa molecular. ${ }^{25}$ Os sinais alargados, provenientes principalmente de macromoléculas, poderiam prejudicar a análise quimiométrica por causarem sobreposição de sinais e com isso diminuir a quantidade de informação contida nos espectros. Por outro lado, a manutenção dos sinais alargados pode representar uma informação importante na distinção entre os diferentes cultivares do feijão. Dessa forma, as medidas espectroscópicas foram também realizadas utilizando-se a ZGCPPR, uma sequência de pré-saturação do sinal da água mas sem a utilização do filtro de $\mathrm{T}_{2}{ }^{26}$

\section{Tratamento dos dados espectroscópicos}

Os espectros de RMN de ${ }^{1} \mathrm{H}$ tiveram as distorções de fases e linhas de base corrigidas pelo programa TopSpin (v 2.1, Bruker Biospin), o qual transformou automaticamente os espectros para arquivos ASCII. Os arquivos ASCII foram importados para o programa Microcal Origin (v 5.0, Microcal Software), onde a matriz gerada foi calibrada tendo o TMS como referência. Após essa etapa a matriz foi importada para o programa Pirouette (v 3.11, Infometrix), para o tratamento multivariado de dados com o método de análise de componentes principais (PCA). Previamente à análise estatística, algumas regiões foram removidas a fim de eliminar qualquer variabilidade na supressão do sinal da água da amostra. Nesse estágio também foram eliminados os sinais dos hidrogênios residuais do TMSP- $d_{4}$, entre outros sinais indesejáveis. A aplicação da PCA permitiu que os dados fossem projetados no espaço definido pelo primeiro plano fatorial, retendo significativa porcentagem de variância acumulada dos dados originais. A análise por agrupamento hierárquico (HCA) foi aplicada para o estudo da similaridade entre os indivíduos (diferentes cultivares/variedades de feijão) com base na distribuição das regiões espectrais pré-selecionadas.

\section{RESULTADOS E DISCUSSÃO}

Os estudos realizados com dois cultivares de seis diferentes variedades de feijão comum desenvolvidos pela Estação Experimental da Embrapa - Arroz e Feijão evidenciaram sinais característicos de compostos fenólicos, tais como antocianinas (hidrogênios aromáticos - $\delta 6,6$ a 9,1 , glicosídicos - $\delta 3,9$ a 5,5 e metoxilas), ${ }^{27}$ flavonoides glicosilados (hidrogênios aromáticos - $\delta 6,4$ a 8,3 e glicosídicos - $\delta 3,5$ a $5,5)^{28}$ e ácidos cinâmicos esterificados com diácidos tetra-hidroxilados (hidrogênios ácidos em $\delta 9,3$, aromáticos e olefínicos - $\delta$ 6,4 a 7,6, hidrogênios carbinólicos dos diácidos - singletos entre $\delta 4,6$ e 4,8 e metoxilas em $\delta 3,8) .{ }^{17}$ A Figura $1 \mathrm{~S}$ (material suplementar) mostra os espectros de RMN de ${ }^{1} \mathrm{H}$ para uma amostra de feijão utilizando-se as sequências de pulsos CPMG e ZGCPPR durante a aquisição dos espectros. Comparando-se esses espectros, pode-se observar que na sequência ZGCPPR alguns sinais aparecem mais amplificados, destacando-se a região dos hidrogênios aromáticos, entre $\delta 6,5$ e 9,3.

Antes de se executar a análise quimiométrica fez-se uma análise visual dos dados, com o objetivo de verificar as diferenças entre os espectros de RMN de ${ }^{1} \mathrm{H}$. Essa análise é importante para verificar se as diferenças espectrais são originárias das composições das amostras ou se estão relacionadas a problemas nas medidas espectroscópicas, tais como o mau ajuste do campo magnético na região próxima à amostra (shimming), a eficiência da pré-saturação do sinal de $\mathrm{H}_{2} \mathrm{O}$ / HDO, a correção de fase e linha de base etc. Esse cuidado é necessário para que variações nesses parâmetros não levem a erros nas análises quimiométricas, pois as diferenças espectrais, características das amostras ou introduzidas através de erros experimentais, levarão à distinção entre as amostras que poderão ser classificadas em grupos diferentes. No conjunto de dados em questão nenhuma amostra apresentou problemas visíveis.

Uma forma de otimizar as análises quimiométricas é, sem dúvida, a seleção das variáveis mais importantes para a discriminação entre os grupos ou classes de amostras. Inicialmente, a análise foi feita incluindo toda a região espectral, com exceção da região dos sinais dos hidrogênios da referência TMSP-d4 e da água $\left(\mathrm{H}_{2} \mathrm{O} / \mathrm{HDO}\right)$, resultando em uma discriminação razoável entre as amostras (dados não apresentados). Entretanto, o uso de todo o espectro acarreta na inclusão de variáveis desnecessárias, como regiões que contenham apenas ruídos eletrônicos, tornando a matriz de dados complexa e demandando maior tempo na execução dos cálculos. Por essa razão, seguiu-se a exclusão das regiões contendo apenas ruídos e repetiu-se a análise por PCA, para as duas sequências de pulsos distintas. Quanto à discriminação dos diferentes cultivares do feijão, observou-se um melhor agrupamento quando se utilizou a sequência de pulsos ZGCPPR e, portanto, essa foi a sequência de pulso utilizada durante toda a análise multivariada.

Os parâmetros utilizados na PCA (Figura 2) que conduziram a uma melhor discriminação entre as amostras de grãos dos diferentes cultivares de feijão foram o autoescalonamento (centrado na média e escalado pela variância) e a normalização dos dados. Para o conjunto de dados contendo os espectros de RMN de ${ }^{1} \mathrm{H}$, obtidos pela sequência de pulsos ZGCPPR, o número ótimo de fatores foi 6 e essas componentes descreveram 84,7\% da variância acumulada dos dados. Já para o conjunto envolvendo os espectros de RMN de ${ }^{1} \mathrm{H}$ obtidos pela sequência CPMG foram necessárias 5 componentes principais, as quais descreveram 50,9\% da variância dos dados. A variância acumulada retida para descrição do primeiro plano fatorial foi de $50 \%$, para a sequência ZGCPPR.

As análises por PCA e HCA mostraram uma tendência de discriminação em quatro grupos (Figura 2). Analisando-se as variáveis de maior peso na discriminação desses grupos e o perfil espectroscópico dos grãos desses cultivares, observou-se que as amostras contidas no grupo localizado na região de escores negativos da PC1 e PC2, ou seja, as amostras 6, 7, 10 e 11, se caracterizam pelos hidrogênios carbinólicos $(\delta 3,5$ a 4,0$)$ e vinílicos $(\delta 5,3$ a 5,0), sugerindo um alto teor de carboidratos e compostos olefínicos. A região de escores negativos para PC1 e positivos para PC2, que contém as amostras $\mathbf{8}$ e $\mathbf{9}$, caracteriza-se por hidrogênios nas faixas $\delta 5,7$ a 6,5 e $\delta 8,0$ a 9,3 , relativos aos compostos aromáticos, flavonoides e antocianinas. Por outro lado, o grupo formado na região de escores positivos, representado pelos cultivares 1 e 12, caracteriza-se pela presença de aminoácidos aromáticos, com base nos hidrogênios na região entre $\delta$ 7,0 e 7,5. Já o grupo formado pela região de escores positivos para PC1 e 


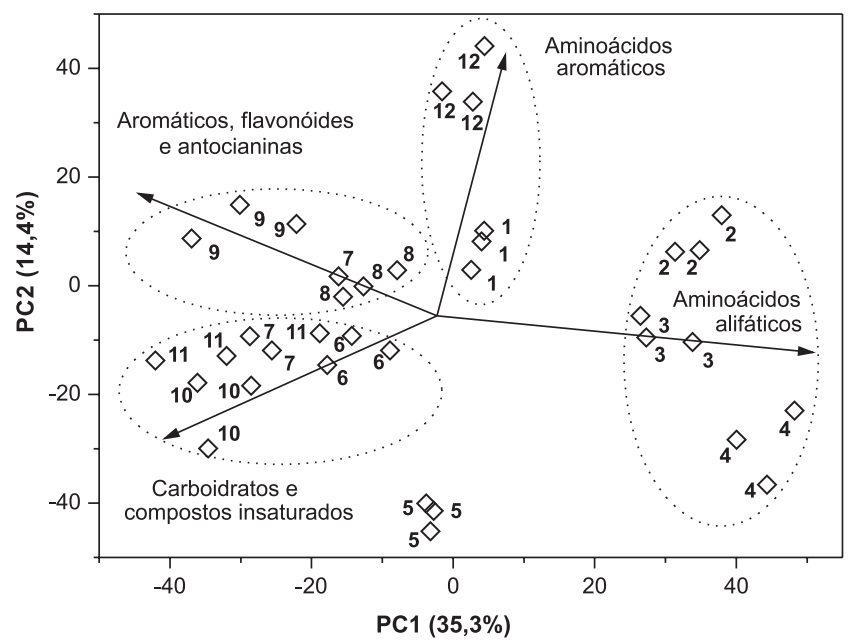

Figura 2. Gráfico dos escores da PC1 x PC2 para os grãos de feijão usando a sequência de pulsos ZGCPPR

negativos para PC2, composta pelos cultivares 2,3 e 4, é rica em aminoácidos alifáticos ( $\delta 2,2$ a 2,5 e $\delta 3,0$ a 3,2). Vale destacar que o feijão campeiro (8) é geneticamente descendente do feijão mulatinho (11), o que justifica a proximidade destes cultivares.

Analisando-se exclusivamente a presença dos oligossacarídeos rafinose, estaquiose e verbascose, observou-se nos espectros de RMN de ${ }^{1} \mathrm{H}$, através dos sinais dos hidrogênios anoméricos da glicose em cada uma das unidades sacarídicas, uma variação considerável nos teores desses açúcares nos diferentes cultivares de $P$. vulgaris (Figura 3). Na análise por PCA, o autoescalonamento dos dados conduziu a uma melhor discriminação entre as amostras de grãos dos diferentes cultivares de feijão. O número ótimo de fatores foi 2 e essas componentes descreveram $76 \%$ da variância acumulada dos dados originais. Nesse sentido, a análise por PCA da região compreendida entre $\delta$ 5,38 e 5,48 discriminou os cultivares Irai (4) e Timbó (10) como aqueles que possuem o maior teor dos oligossacarídeos estaquiose e verbascose e o cultivar Pérola (5) como o que possui maior teor relativo de rafinose (Figura 4).
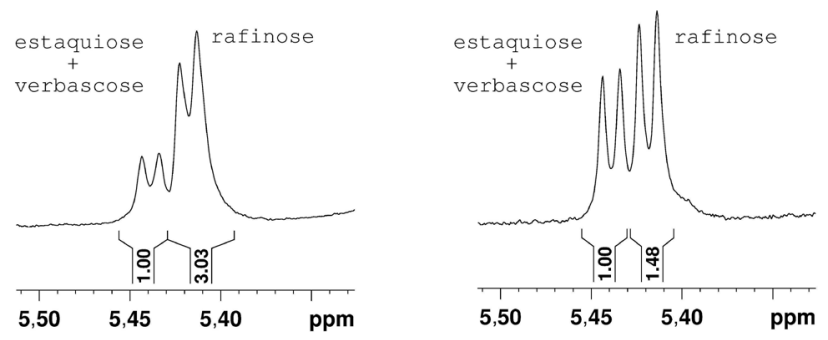

Figura 3. Região dos hidrogênios anoméricos da estaquiose, verbascose e rafinose para os cultivares 1 e 4

\section{CONCLUSÕES}

A ressonância magnética nuclear, através da técnica HR-MAS, aliada aos métodos quimiométricos, mostrou ser uma ferramenta capaz de separar os diferentes cultivares do feijão comum, Phaseolus vulgaris, em quatro grupos distintos de acordo com a sua composição química. Permitiu ainda a identificação dos cultivares que possuem o maior teor relativo dos oligossacarídeos rafinose, verbascose e estaquiose. Todas as análises foram realizadas sem a necessidade de se utilizar quaisquer métodos de pré-tratamento ou extração das amostras. Além disso, pode-se observar pela análise dos

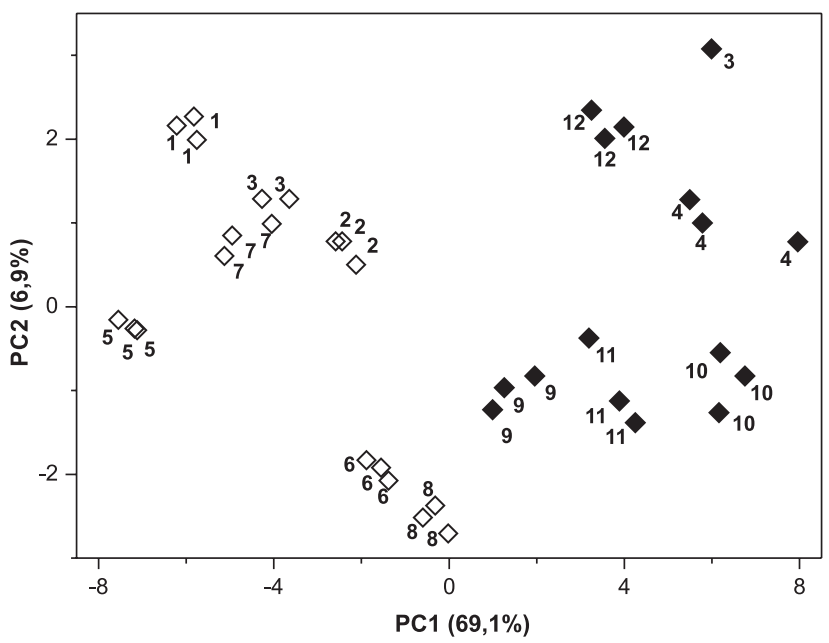

Figura 4. Gráfico dos escores de PC1 x PC2 na região dos hidrogênios anoméricos, com predomínio da estaquiose e verbascose $(\diamond)$ ou rafinose $(\diamond)$, usando a sequência de pulsos ZGCPPR

vários espectros que o melhor resultado é obtido quando se utiliza tanto a sonda quanto os rotores específicos para análise de material semi-sólido (heterogêneo). Os espectros são muito semelhantes, no que diz respeito à resolução espectral, aos obtidos para soluções.

\section{MATERIAL SUPLEMENTAR}

A Figura $1 \mathrm{~S}$ referente aos espectros de $\mathrm{RMN}$ de ${ }^{1} \mathrm{H}$ para uma amostra de feijão utilizando-se as sequências de pulsos CPMG e ZGCPPR durante a aquisição dos espectros, está disponível em http:// quimica-nova.sbq.org.br, na forma de arquivo PDF, com acesso livre.

\section{AGRADECIMENTOS}

À FAPESP e ao CNPq pelo apoio financeiro. Ao CNPq pela bolsa de pós-doutoramento de L. M. Lião e de Iniciação Científica do aluno P. P. A. Cavalcante.

\section{REFERÊNCIAS}

1. Aguiar, M. R. M. P.; Gemal, A. L.; San Gil, R. A. S.; Quim. Nova 1999, $22,553$.

2. Gil, A. M.; Alberti, E.; Belton, P. S.; Humpfer, E.; Spraul, M.; Magn. Reson. Chem. 1997, 35, S101.

3. Gil, A. M.; Duarte, I. F.; Delgadillo, I.; Colquhoun, I. J.; Cascuscelli. F.; Humpfer, E.; Spraul, M.; J. Agric. Food Chem. 2000, 48, 1524; Belton, P. S.; Delgadilho, I.; Gil, A. M.; Casuscelli, F.; Colquhoun, I. J.; Dennis, M. J.; Spraul, M.; Magn. Reson. Chem. 1997, 35, S52.

4. Wang, Y.; Bollard, M. E.; Keun, H.; Antii, H.; Beckonert, O.; Ebbels, T. M.; Lindon, J. C.; Holmes, E.; Tang, H.; Nicholson, J. K.; Anal. Biochem. 2003, 323, 26.

5. Händel, H.; Gesele, E.; Gottschall, G.; Albert, K.; Angew. Chem., Int. Ed. 2003, 42, 438.

6. Pampel, A.; Fernandez, M.; Freude, D.; Kärger, J.; Chem. Phys. Lett. 2005, 407, 53; Roland, J.; Michel, D.; Magn. Reson. Chem. 2000, 38, 587.

7. Schröder, H.; Comb. Chem. High Throughput Screening 2003, 6, 741.

8. Franks, W. T.; Wylie, B. J.; Schimidt, H. L. F.; Nieuwkoop, A. J.; Mayrhofer, R. -M.; Shah, G. J.; Graesser, D. T.; Rienstra, C. M.; Proc. Natl. Acad. Sci. U.S.A. 2008, 105, 4621.

9. Blaise, B. J.; Giacomotto, J.; Elena, B.; Dumas, M. -E.; Toulhoat, P.; Sagalat, L.; Emsley, L.; Proc. Natl. Acad. Sci. U.S.A. 2007, 104, 19808. 
10. Polito, L.; Colombo, M.; Monti, D.; Melato, S.; Caneva, E.; Prosperi, D.; J. Am. Chem. Soc. 2008, 130, 12712.

11. Alcantara, G. B.; Honda, N. K.; Ferreira, M. M. C.; Ferreira, A. G.; Anal. Chim. Acta 2007, 595, 3.

12. Brescia, M. A.; Jambrenghi, A. C.; Martino, V.; Sacco, D.; Giannico, F.; Vonghia, G.; Sacco, A.; Ital. J. Anim. Sci. 2002, 1, 151; Brescia, M. A.; Sgaramella, A.; Ghelli, S.; Sacco, A.; J. Sci. Food Agric. 2003, 83, 1463.

13. Sacco, A.; Bolsi, I. N.; Massini, R.; Spraul, M.; Humpfer, E.; Ghelli, S.; J. Agric. Food Chem. 1998, 46, 4242.

14. Shintu, L.; Caldarelli, S.; J. Agric. Food Chem. 2005, 53, 4026.

15. Monteiro, M. R.; Ambrozin, A. R. P.; Santos, M. S.; Boffo, E. F.; Pereira-Filho, E. R.; Lião, L. M.; Ferreira, A. G.; Talanta 2009, 78, 660; Monteiro, M. R.; Ambrozin, A. R. P.; Lião, L. M.; Boffo, E. F.; Tavares, L. A.; Ferreira, M. M. C.; Ferreira, A. G.; Energy Fuels 2009, 23, 272.

16. http://www.ibge.gov.br, acessada em Julho 2008.

17. Lin, L. Z.; Harnly, J. M.; Pastor-Corrales, M. S.; Luthria, D. L.; Food Chem. 2008, 107, 399.

18. http://www.anvisa.gov.br, acessada em Julho 2008
19. Anderson, J. W.; Smith, B. M.; Washnock, C. S.; Am. J. Clin. Nutr. 1999, $70,464$.

20. Viswanathan, M.; Ramachandran, A.; Indira, P.; Snehalatha, C.; Mohan, V.; Kymal, P. K.; Nutr. Rep. Int. 1989, 40, 803.

21. Leathwood, P.; Pollet, R.; Appetite 1988, 10, 1.

22. Hangen, L.; Bennink, M. R.; J. Nutr. Cancer 2002, 44, 60.

23. Gibson G. R.; Roberfroid, M. B.; J. Nutr. 1995, 125,1401.

24. Anisha, G. S.; Prema, P.; Food Chem. 2008, 106, 1175.

25. Claridge, T. D. W. Em High-Resolution NMR Techniques in Organic Chemistry; Tetrahedron Organic Chemistry Series; Baldwin, J. E.; Willians, R. M., eds.; Elsevier: Oxford, 1999, vol. 19.

26. Derome, A. E. Em Modern NMR Techniques for Chemistry Research; Tetrahedron Organic Chemistry Series; Baldwin, J. E.; Magnus, P. D., eds.; Pergamon Press: Oxford, 1987, vol. 6.

27. Kosir, I. J.; Kidric, J.; An. Chim. Acta 2002, 458, 77.

28. Dinelli, G.; Bonetti, A.; Minelli, M.; Marotti, I.; Catizone, P.; Mazzanti, A.; Food Chem. 2006, 99, 105. 
PERFIL QUÍMICO DE CULTIVARES DE FEIJÃO (Phaseolus vulgaris) PELA TÉCNICA DE HIGH RESOLUTION MAGIC ANGLE SPINNING (HR-MAS)

Luciano Morais Lião*, Rafael Choze, Pedro Paulo Araujo Cavalcante, Suzana da Costa Santos e Pedro Henrique Ferri Instituto de Química, Universidade Federal de Goiás, CP 131, 74001-970 Goiânia - GO, Brasil Antonio Gilberto Ferreira
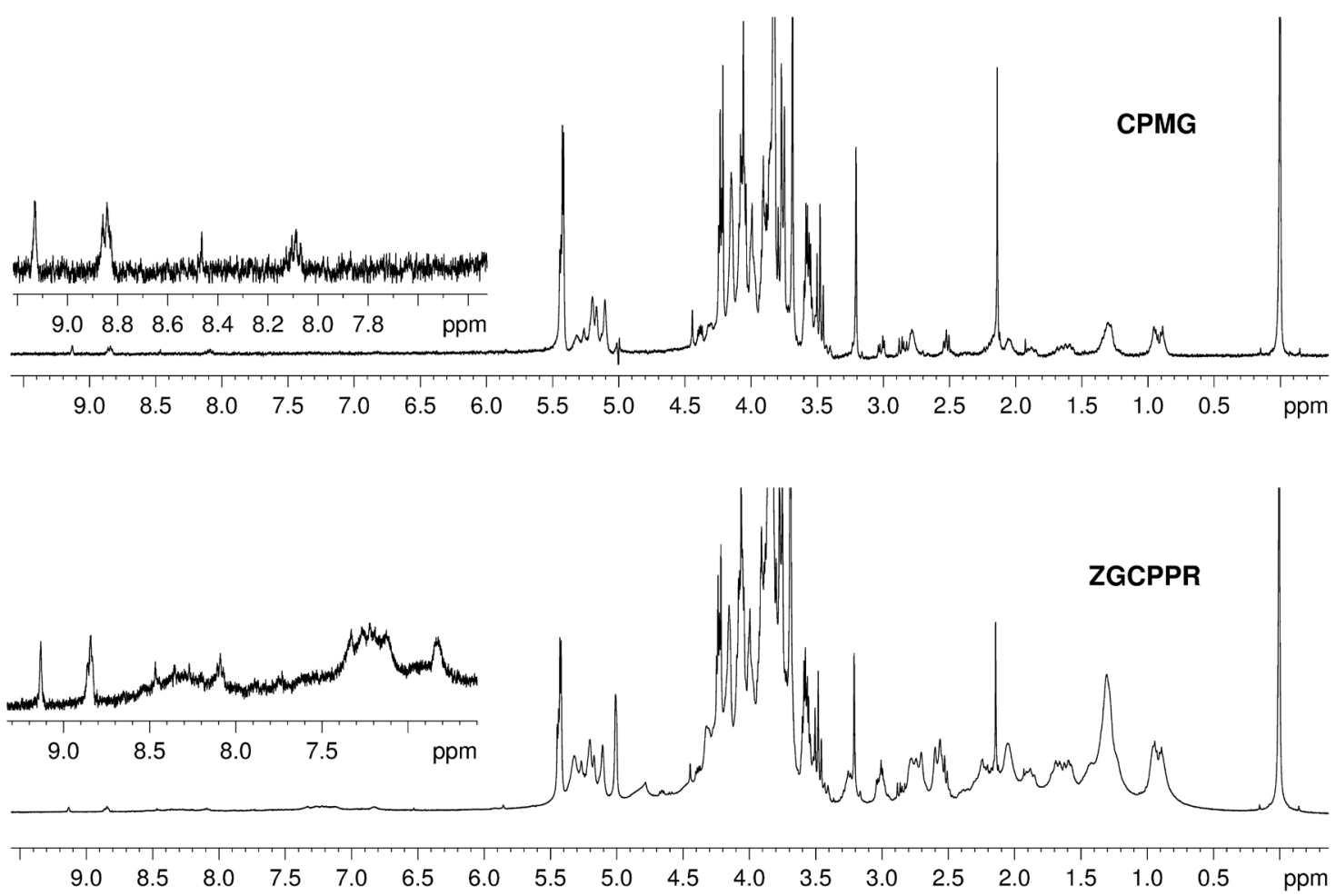

Figura 1S. Espectros de RMN de ${ }^{1} \mathrm{H}$ de feijões adquiridos na sonda HR-MAS utilizando as sequências de pulsos CPMG modificada e ZGCPPR, com ampliação da região de $\delta$ 6,5 a 9,2

*e-mail: luciano@ quimica.ufg.br 